\title{
Post-publication promotion in rheumatology: a survey focusing on social media
}

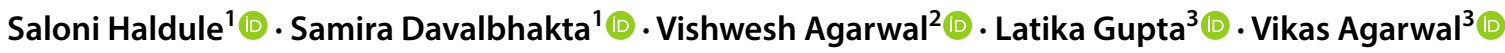

Received: 12 August 2020 / Accepted: 1 September 2020 / Published online: 13 September 2020

๑) Springer-Verlag GmbH Germany, part of Springer Nature 2020

\begin{abstract}
The use of social media platforms (SMPs) in the field of scientific literature is a new and evolving realm. The past few years have seen many novel strategies to promote engagement of readers with articles. The aim of this study was to gauge the acceptance, opinion, and willingness to partake in the creation of online social media educative material among authors. We conducted a validated and anonymized cross-sectional e-survey with purposive sampling among authors of the Indian Journal of Rheumatology journal over a cloud-based platform (SurveyMonkey). Descriptive statistics are used and values expressed as the number of respondents ( $n$ ) against each answer. Of 408 authors, 102 responded. We found that a large majority (74) supported promotions on SMPs. Visual abstracts (81) were the most preferred means for promotion. A reasonable proportion (54) of the authors held the view that they could make these materials for themselves, with little guidance. However, currently only a few (47) were doing so. Awareness on social media editors in rheumatology was dismal (4). Citations were the preferred metric of article visibility (95), followed by altmetrics (21). These findings suggest that authors support article promotions on SMPs, although most do not promote their articles. Graphical abstracts are the preferred means of promotions. Further, the opinion on logistics is divided, calling for larger studies to understand the factors that need to be addressed to bridge the gap.
\end{abstract}

Keywords Social media $\cdot$ Information dissemination $\cdot$ Publications $\cdot$ Artificial intelligence $\cdot$ Rheumatology $\cdot$ Scholarly communication

Latika Gupta

drlatikagupta@gmail.com

Saloni Haldule

saloni.hal@gmail.com

Samira Davalbhakta

samdavy98@gmail.com

Vishwesh Agarwal

vishwesh002@gmail.com

Vikas Agarwal

vikasagr@yahoo.com

1 Byramjee Jeejeebhoy Government Medical College and Sassoon General Hospitals, Pune, India

2 Mahatma Gandhi Mission Medical College, Navi Mumbai, India

3 Department Clinical Immunology and Rheumatology, Sanjay Gandhi Postgraduate Institute of Medical Sciences, Lucknow, India

\section{Introduction}

Social media is a broad term that encompasses the use of technology to participate in social networking. The use has extended in the twenty-first century beyond personal networking, to academia and telehealth [1]. The widespread lockdowns and public mandate for social distancing during the ongoing pandemic have further strengthened the case for using social media platforms (SMPs) for academic communication in this period [2]. Several journals have picked up on this trend by sharing their work on SMPs [3].

SMPs are increasingly being used to share information from primary as well as secondary, tertiary, and grey literature $[4,5]$. Of these, original research (primary and secondary articles), comprises the most important updated information for doctors, researchers, and administrators alike [6]. In times when pre-prints are being archived to rapidly disseminate scientific research, articles published in scholarly journals after an extensive peer-review are of greater scientific credence $[7,8]$. The rapidly rising number of research 
articles on an area of interest, such as COVID-19, can make it challenging to assimilate the required information $[9,10]$.

The large volume of information available can be overwhelming and may sometimes hinder the process of learning. Furthermore, retention of information may be hampered by the use of only one type of traditional learning cue, i.e., text [11]. The use of newer methods to present information, such as visual (or graphical, Fig. 1), voice, or video abstracts, can be helpful to attract and sustain the reader's attention. Experts suggest that we retain $10 \%$ of what we read, $20 \%$ of what we hear, and $30 \%$ of what we see [12]. Hence, using additional tools to promote literature on SMPs may bring a dynamicity to the process. Moreover, mixed sensory cues may enhance the learning experience and overcome barriers to poor memory and recall [11]. Infographics, video abstracts and voice abstracts (or podcasts) are potential strategies to enhance the scientific readership experience.

Since these strategies are novel, their implementation comes with challenges of its own, such as an evident lack of clarity as to who should prepare these promotional educative materials. While the authors may be willing to take responsibility themselves, developing such resources is a time-consuming skill, and they may need assistance from trained personnel. Moreover, cost constraints may limit the use of professional editing agencies, more so in the developing countries. Thus, we aimed at studying the acceptance, opinion, and willingness to participate in the creation of online social media educative material among authors of published self-articles in scholarly journals.

\section{Methods}

The e-survey was designed on an online cloud-based website (Survey Monkey ${ }^{\circledR}$.com) with the intent to cover different aspects of social media editing, such as willingness for social media promotions of (2), means of promotion (4), ethics (3), logistics (3), preference for article metrics, publication models and pre-print archiving (2), current knowledge/use of social media for these purposes (4).

\section{Survey design}

The questionnaire featured 22 questions, most (12) of which were multiple choice questions needing a single answer option, while others (9) could have more than one answer option selected, and some (1) needed a single answer to be selected from a list. Four items identified the respondent characteristics, and the rest (18) covered various domains listed above. Choices were closed ended for most (11), with an 'other (please specify)' option where deemed appropriate (11).

Two rheumatologists and three undergraduate medical students reviewed the questions and confirmed them to be representative of the content and face validity. The survey underwent three rounds of dummy fill-ups to identify errors in wording, grammar, and syntax. The respondents could change the answers before submission but not after it. The survey was partly anonymised with Internet Protocol (IP) address and emails of respondents being the only linked identifiers. All questions were made mandatory, such that

\section{Post-publication promotion in rheumatology: do authors believe in social media promotions?}

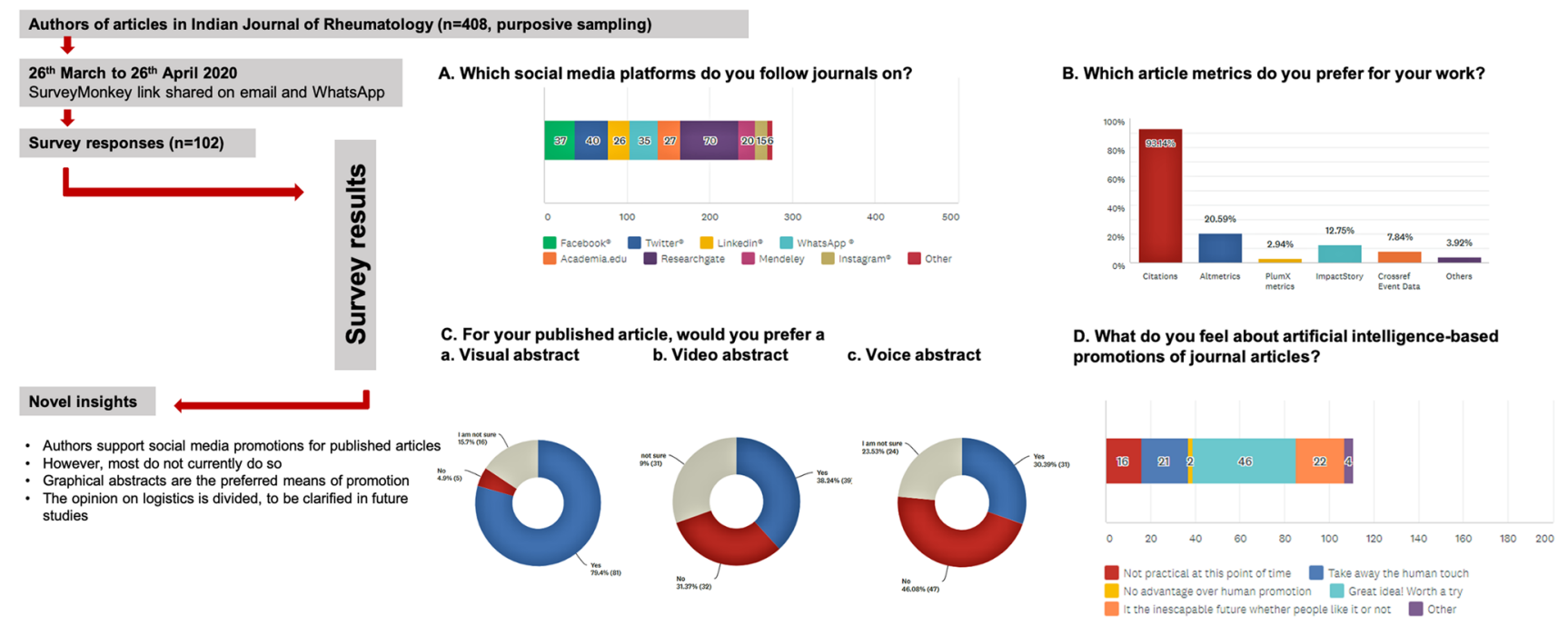

Fig. 1 Infographic demonstrating opinion of authors on post-publication promotion of articles in rheumatology journals 
partial responses were automatically discarded by the SurveyMonkey platform.

\section{Sampling strategy}

Emails of corresponding authors of articles published from 2010 to 2020 in the Indian Journal of Rheumatology (IJR, $n=408$ ) were obtained from Scopus. The IJR is a Scopus and Web of Science indexed platinum open-access society journal of the Indian Rheumatology Association with a wide readership in India. The questionnaire was circulated to the list hence obtained. The eligible participants were given a month's time to voluntarily complete the survey from 26 March to 26 April 2020. The survey link was open from the time the authors were first intimated about the study. The cover letter included details on the background and purpose of the study. Informed consent was taken at the beginning of the survey and no incentives offered for survey completion.

\section{Data handling and confidentiality}

Internet Protocol (IP) address checks were done to avoid duplicated responses from a single respondent. Data handling was completely anonymous, with the IP addresses and email lists remaining with the first and corresponding author. Other authors had access to the synthesized data in tables without linked identifiers.

An exemption from review was obtained from the institute ethics committee of Sanjay Gandhi Post Graduate Institute Of Medical Sciences, Lucknow as per local guidelines. We adhered to the Checklist for Reporting Results of Internet E-surveys to report the data [13].

\section{Statistical analysis}

Descriptive statistics were used, and figures designed using the SurveyMonkey website. Data are expressed as median, percentage and inter-quartile range. Numbers in brackets signify the number of respondents for the answer choices being discussed.

\section{Results}

Of the 102 respondents, most (83) lived in India and were practicing rheumatology (77, Table 1$)$. Nearly half (50) were in practice for 11 years or more. The average survey time was $5 \mathrm{~min}$ and $4 \mathrm{~s}$. The response rate was $25 \%$.

\section{Social media promotions and platforms}

Over two-thirds (74) said they would like their publication promoted on social media, ResearchGate (70) being the most preferred platform, followed by Twitter (40), Facebook (37), WhatsApp (35), Academia.edu (27), and LinkedIn (26). Only five said they would not prefer their publication being promoted.

However, only 47 promoted their articles [on ResearchGate followed by WhatsApp (32)] currently, and 26 did not do so. When asked who should promote articles, specially appointed social media editors were preferred by nearly half (46) while 24 felt all editors should do so, and 29 believed only the copyright holders can promote their publication on social media.

Most felt the use of appropriate hashtags (43) and appropriate timing (42) were crucial for successful promotion, and an equal proportion (46) advocated the use of artificial intelligence-based algorithms for the same. A dismal four people knew the correct number of rheumatology journals that have social media editors. Nearly three-fourths (77) were unaware that the IJR had SME (s).

\section{Ancillary abstracts}

Visual (or graphical) abstracts were the most preferred (81) means for enhancing visibility among authors, while voice (47) and video (39) abstracts were preferred by fewer. While 54 felt they could prepare a visual abstract by guidance from the editorial team, another 25 felt the editorial team should do it (without charges). On the other hand, 34 felt they could do video abstracts themselves and another 37 said it should be outsourced to a third party. Similar proportions (36) were willing to prepare voice abstracts after guidance, though outsourcing was less supported (11).

\section{Publication models}

Preference for citations as metric (95) was almost unanimous though nearly one in five (21) also preferred Altmetrics for visibility. The Green open access was the most preferred model (45) followed closely by Platinum Open Access (31). Pre-Print Archiving was preferred by a minority (15).

\section{Discussion}

We noted good acceptance for social media promotions of published scholarly literature by over two-thirds of the authors, although less than half had actually done so. Visual (or graphical) abstracts were the most preferred means for enhancing visibility among authors. However, the opinion on logistics of promotion and preparation was divided.

The use of visual abstracts was popular amongst the respondents. However, video and voice abstracts were far less so. This could possibly be, in part due to the perception that making visual abstracts is simpler than the other 
Table 1 Survey responses

Social media usage

Question

Response

Do you follow any medical journals on social media?

Yes

$60(58.8 \%)$

No

$42(41.1 \%)$

Would you like your publication being promoted on social media?

Yes

$74(72.5 \%)$

No

$10(9.8 \%)$

Not sure

$18(17.6 \%)$

Which social media platforms would you prefer to follow journals on?

Facebook

$37(36.2 \%)$

Twitter

$40(39.2 \%)$

LinkedIn

$26(25.4 \%)$

WhatsApp

35 (34.3\%)

Academia.edu

27 (26.4\%)

Researchgate

$70(68.6 \%)$

Mendeley

$20(19.6 \%)$

Instagram

$15(14.7 \%)$

Other

None

$5(4.9 \%)$

Doximity

$1(0.9 \%)$

Do you promote your article(s) on any of the following social media sites?

Facebook

$16(15.6 \%)$

Twitter

$13(12.7 \%)$

LinkedIn

$9(8.8 \%)$

WhatsApp

$32(31.3 \%)$

Academia.edu

$9(8.8 \%)$

Researchgate

$47(46.1 \%)$

Mendeley

$10(9.8 \%)$

Instagram

$2(1.9 \%)$

Other

$26(25.5 \%)$

None

$26(25.5 \%)$

Which model of article sharing would you prefer the most (single answer)?

Pre-print archiving

Green open access: authors can self-archive copies on social media or university sites

$45(44.1 \%)$

Gold open access: journals allow open access to all readers on payment of an APC (article processing charge)

$11(10.7 \%)$

Platinum Open access: journals allow open access to all readers without open access (funds for publication are usually provided

$31(30.4 \%)$

by an university or society)

If yes, who do you think should prepare the visual abstract? (multiple options)

You yourself

$54(52.9 \%)$

You with guidance from the journal editorial team

Editorial team (willing to pay for it)

Editorial team (not willing to pay for it)

Outsource to third party

None of the above

I am not sure

Not applicable

If yes, who do you think should prepare the video abstract? (multiple options)

You yourself

You with guidance from the journal editorial team 
Table 1 (continued)

Social media usage

Question

Response

Editorial team (willing to pay for it)

$4(3.9 \%)$

Editorial team (not willing to pay for it)

$19(18.6 \%)$

Outsource to third party

$7(6.8 \%)$

None of the above (Specify)

$37(36.2 \%)$

Who should promote articles on social media for a journal?

All editors

$24(23.5 \%)$

Specially appointed social media editor

$46(45.1 \%)$

Automated bot

$10(9.8 \%)$

Authors

$18(17.6 \%)$

All of the above

$32(31.3 \%)$

Other

$5(4.9 \%)$

None

$3(2.9 \%)$

I am not sure

$2(1.9 \%)$

If yes, who do you think should prepare the voice abstract? (multiple options)

You yourself

$19(18.6 \%)$

You with guidance from the journal editorial team

$36(35.2 \%)$

Editorial team (willing to pay for it)

$4(3.9 \%)$

Editorial team (not willing to pay for it)

$28(27.4 \%)$

Outsource to third party

$11(10.7 \%)$

Other

$20(19.6 \%)$

No

$16(15.6 \%)$

Not sure

Not applicable

What do you feel about promotion of your article on social media?

Only the copyright holders can promote

$29(28.4 \%)$

Only the journal editors should promote

$24(23.5 \%)$

Only authors should promote

$10(9.8 \%)$

It is unethical to promote articles on social media

$8(7.8 \%)$

Anyone can promote

$28(27.4 \%)$

Other

Uncertain of benefit if message is lost

The copyright holders and authors can promote

Copy right holders and authors for platinum model

$1(0.9 \%)$

What strategies are you aware of for promoting your article on social media?

Optimal timing of update

$42(41.1 \%)$

Appropriate hashtag use

$43(42.1 \%)$

Tagging important stakeholders

$28(27.4 \%)$

Using bots

$8(7.8 \%)$

Using dedicated software

$16(15.6 \%)$

Paid promotion

$10(9.8 \%)$

None of the above

$33(32.3 \%)$

Other

$0(0 \%)$

How many rheumatology journals having a social media editor or editors are you aware of?

$<5$

$31(30.3 \%)$

5-10

$12(11.7 \%)$

11-20

$4(3.9 \%)$

21-40

$1(0.9 \%)$

$>41$ 
Table 1 (continued)

\begin{tabular}{lc}
\hline Social media usage & Response \\
\hline Question & $1(0.9 \%)$ \\
\hline I am not sure & $25(24.5 \%)$ \\
Does the Indian Journal of Rheuamtology have social media editor(s)? & $5(4.9 \%)$ \\
Yes & $72(70.5 \%)$ \\
No & $70.5 \%$ \\
I am not aware & \\
\hline
\end{tabular}

options. This is supported by our finding that over half felt they could themselves make the visual abstracts with due guidance, as opposed to one-third who felt the same regarding video and voice abstracts. However, further studies should be done to determine the reasons for preferences within the various promotional tools, as well to understand who would be best suitable to prepare them.

Visual abstracts are a clear, concise format to expeditiously disseminate information, and are now increasingly being encouraged by various journals [14]. At times where healthcare workers are burdened with medical care of Covid19 patients, visual abstracts allow them to find the most relevant material quickly. A recent study showed that infographics summarized medical research literature and were associated with higher reader preference and lower cognitive load during summary review, although no difference was found in late information retention [15].

In the current fast-paced world, where technology governs most aspects of development, scientific literature must evolve to fit the needs of the hour. Now more than ever, as we fight a global pandemic, information needs to travel faster, and beyond any man-made borders to be effective. SMPs are a fitting solution for many of these needs. It has been increasingly found that there exists a relationship between the traditional impact factor and activity on social media, with some sources having a near perfect correlation [16]. However, many researchers feel overwhelmed by the internet and social media due to a lack of scientific guidance [17]. Moreover, over $90 \%$ professionals use social media for personal reasons, with merely $65 \%$ doing so for academics purposes [18].

While citations are the traditionally preferred metric of visibility, altmetrics are now increasingly being identified as an important metric for publication success [19]. Altmetrics are non-traditional article-level bibliometrics that gather details of engagement of research work on a wide variety of online platforms, including but not limited to mentions in the news, blogs, and on Twitter and other SMPs, article pageviews and downloads, etc. A drawback of using citations is the inevitable time lag in acquiring them, reducing utility for young scholars. Moreover, citations reflect article use by researchers. But in this shrinking world with borderless communication, and there are many more stakeholders than there were before. It is just as important for hospital administrators, policy makers, clinicians, and educators to be informed of the latest developments, and to modify their practices accordingly. Altmetrics may provide a more wholesome picture of the article visibility and utility from diverse areas. Moreover, altmetric scores on researcher platforms, such as academia and Mendeley, may translate into higher cite scores, due to the characteristics of the readership population [20]. A recent study found a high correlation (0.83) between the Scimago Journal Rank scores and the number of followers on Twitter, despite adjusting for time since creation of the account, further substantiating enhanced visibility of articles using SMPs and the potential to enhance journal (and consequently article) metrics with social media practices [16].

Acceptance of social media promotions was good among our respondents, although understanding of its utility may be poor, translating to the admittedly low rates of active social media practice for academic promotions. The use of SMPs for promotions of own articles was inferred as practice. This may be improved by educating healthcare professionals about the various aspects of social media use [21]. Notably, the preferred platforms for promotions may vary, with ResearchGate and WhatsApp being popular in India and WeChat in China [22]. A recent survey conducted among rheumatologists in the European network recorded Facebook as the most commonly use platform, though only $46 \%$ used it to establish a professional online presence [23]. Further research may provide a better understanding of individual differences.

Interestingly, the opinion regarding the responsibility of social media promotions was divided among authors. Though nearly half felt social media editors (SMEs) should do so, a significant number felt only editors and copyright holders should take the responsibility. Social media promotions require specific skills, time and dedication. It is also essential to be aware of standards for quality and content for effective promotions. SMEs are more likely to be aware of these, and the various tools to meet these requirements. A lack of awareness regarding availability of SMEs for rheumatology journals was evident in our respondent population, 
possibly a reflection of poor interest and interaction of researchers on SMPs. A shift in the culture from researchers being passive bystanders to active participants in this process may enhance academic engagement [24].

Although SMEs may be proficient at promotions, every author and member of the scientific community can, and should, partake in the narrative themselves. The impressions of tweets are shown to improve with a wider retweeting network. The uses of hashtags and mentions are other effective ways to enhance engagement [25]. Audio abstracts may feature as podcasts, which are popular in the West, although the trend may not have caught on so well in India yet. Podcasts are increasingly being used for medical education, both within teaching institutions and on an international scale by major journals.[26]. Nearly half of our respondents were open to audio abstracts, suggesting this merits exploration in India as well.

Copyright and plagiarism remain an important concern with social media promotions. When sharing images and infographics, it is essential to check copyright permissions, and provide the source information. Some journals allow certain articles to be freely shared for promotions. Articles shared on open-access media are known to have better visibility, and this was reflected in our respondents' opinion too.

Our findings show that pre-print archiving was preferred by less than one-third of the respondents. Preprint servers like MedRxiv are rapidly gaining popularity, especially since the Covid-19 pandemic [27]. Even though most of these submissions on archival platforms do not undergo the process of peer review, there has been a boom in literature available through MedRxiv/Bioarxiv. Most journals too, now encourage pre-print submissions. However, resorting to such information for key decisions can lead to disastrous consequences.

The authors agreed that the use of artificial intelligence algorithms to promote articles would be a beneficial tool. Algorithms, such as the MedFact, which was published in the Canadian Conference on Artificial Intelligence in 2018, aim at enabling recommendations of trusted medical information within health-related social media discussions [28]. MedFact automatically extracts relevant keywords from online discussions and queries trusted medical literature with the aim of embedding related factual information into the discussion [28]. Extrapolated to medical literature, this may be one of the solutions to the ethical concerns regarding social media and its lack of credible information. However, social media is a double-edged sword, and it is vital to educate professionals regarding ethical use to deliver credible scientific information and perspectives without engaging in misinformation, data ownership violation, breach of personal privacy, incivility, cyber bullying, and professional misconduct [29]. This is essential as the work of promoting articles on SMPs is a trinomial; an amalgamation of technological challenges with ethics in science and dealing with widely varying cultural diversity [30]. Moreover, it is important to be mindful of the fact that use of SMPs has addictive potential, and although excessive preoccupation correlates with task-related and relationship-building behaviours, it contributes most strongly to negative social media-related deviant behaviour at the workplace. Fortunately, individuals using SMPs for academic growth are aware of positive as well as negative effects [31], and thus, reinforcement of this knowledge may potentially curb negative behaviours related to social media use.

While our survey is limited by self-selection and recall biases, and unknown characteristics of the non-respondents, it highlights key issues regarding the role of social media in academia, while laying the groundwork for larger studies for understanding these further. The responses were limited in the current study by a short survey duration, coinciding with the onset of the Covid-19 pandemic. Our anonymized survey did not allow correlation of authors' responses with their publication activity. However, we hope that the primary insights offered by this exploratory study would pave the way for larger global study across non-Indian rheumatology journals on the subject.

\section{Conclusion}

To conclude, authors in rheumatology journal support the use of social media for promotions of published scholarly literature, although this does not translate into practice. The use of graphical abstracts is supported by a majority, with video and voice abstracts being less popular. The opinion on logistics is divided, calling for larger studies to understand the factors that need to be addressed to bridge the gap.

Acknowledgements The authors thank all respondents for filling the questionnaire and Dr Sakir Ahmed for reviewing the survey questions.

Author contributions All authors were involved in ideation, data collection and manuscript preparation. All agree with the submitted version of the manuscript, take responsibility for the content of the entire manuscript, and affirm that any queries related to any aspect of the same are appropriately managed.

Funding This study was not funded.

Data availability All data pertaining to the study is included in the manuscript and as supplementary material.

\section{Compliance with ethical standards}

Conflict of interest LG is the social media editor (SME) of the Journal of Clinical Rheumatology and the Indian Journal of Rheumatology. VA is the editor in chief of the Indian Journal of Rheumatology. The 
authors declare that there is no conflict of interest relevant to the manuscript.

Ethical approval An exemption from review was obtained from the institute ethics committee of Sanjay Gandhi Postgraduate Institute of Medical Sciences, Lucknow, India as per local guidelines (2020-110-IP-EXP-16).

Consent to participate The survey was completely anonymised, and informed consent taken from the respondents at the beginning of the exercise.

Consent for publication Informed consent was taken from the respondents at the beginning of the exercise for publication of the results.

\section{References}

1. Trueger NS (2018) Medical journals in the age of ubiquitous social media. J Am Coll Radiol JACR 15:173-176. https://doi. org/10.1016/j.jacr.2017.09.036

2. Pollett S, Rivers C (2020) Social media and the new world of scientific communication during the COVID19 pandemic. Clin Infect Dis. https://doi.org/10.1093/cid/ciaa553

3. Weingart SD, Faust JS (2014) Future evolution of traditional journals and social media medical education. Emerg Med Australas 26:62-66. https://doi.org/10.1111/1742-6723.12192

4. Adams J, Hillier-Brown FC, Moore HJ et al (2016) Searching and synthesising 'grey literature' and 'grey information' in public health: critical reflections on three case studies. Syst Rev 5:164. https://doi. org/10.1186/s13643-016-0337-y

5. Breland JY, Quintiliani LM, Schneider KL et al (2017) Social media as a tool to increase the impact of public health research. Am J Public Health 107:1890-1891. https://doi.org/10.2105/ AJPH.2017.304098

6. Librarians HS LibGuides: Primary vs Secondary Literature in the Biomedical Sciences: Primary vs Secondary Sources. https://libgu ides.library.drexel.edu/c.php?g=859569\&p=6159103. Accessed 3 Aug 2020

7. Rochwerg B, Parke R, Murthy S et al (2020) Misinformation during the coronavirus disease 2019 outbreak: how knowledge emerges from noise. Crit Care Explor 2:e0098. https://doi.org/10.1097/ CCE.0000000000000098

8. Gupta L, Gasparyan AY, Misra DP et al (2020) Information and misinformation on COVID-19: a cross-sectional survey study. J Korean Med Sci. https://doi.org/10.3346/jkms.2020.35.e256

9. Goel A, Gupta L (2020) Social media in the times of COVID-19. JCR J Clin Rheumatol. https://doi.org/10.1097/RHU.0000000000 001508

10. Gupta L, Misra DP, Agarwal V et al (2020) Management of rheumatic diseases in the time of covid-19 pandemic: perspectives of rheumatology practitioners from India. Ann Rheum Dis. https://doi. org/10.1136/annrheumdis-2020-217509

11. The Impact of Presentation Style on the Retention of Online Health Information: a Randomized-Controlled Experiment: Health Communication: 28(3). https://www.tandfonline.com/doi/ abs/10.1080/10410236.2012.683387. Accessed 3 Aug 2020

12. Gloviczki P, Lawrence PF (2018) Visual abstracts bring key message of scientific research. J Vasc Surg 67:1319-1320. https://doi. org/10.1016/j.jvs.2018.04.003

13. Eysenbach G (2004) Improving the quality of web surveys: the checklist for reporting results of internet E-surveys (CHERRIES). $\mathrm{J}$ Med Internet Res 6:e34. https://doi.org/10.2196/jmir.6.3.e34

14. Ibrahim AM, Bradley SM (2017) Adoption of visual abstracts at circulation CQO: why and how we're doing it. Circ Cardiovasc Qual Outcomes. https://doi.org/10.1161/CIRCOUTCOMES.117.003684
15. Martin LJ, Turnquist A, Groot B et al (2019) Exploring the role of infographics for summarizing medical literature. Health Prof Educ 5:48-57. https://doi.org/10.1016/j.hpe.2018.03.005

16. Ordoñez-Gutiérrez JA, Oviedo-Moreno JM, Patino-Hernandez D, Fernández-Ávila DG (2020) Immunology and social networks: an approach towards impact assessment. Rheumatol Int 40:251-256. https://doi.org/10.1007/s00296-019-04459-6

17. Bik HM, Goldstein MC (2013) An Introduction to Social Media for Scientists. PLOS Biol 11:e1001535. https://doi.org/10.1371/journ al.pbio. 1001535

18. Ventola CL (2014) Social media and health care professionals: benefits, risks, and best practices. P T Peer-Rev J Formul Manag 39:491-520 (PMCID: PMC4103576)

19. Sud P, Thelwall M (2014) Evaluating altmetrics. Scientometrics 98:1131-1143. https://doi.org/10.1007/s11192-013-1117-2

20. Mendeley readership altmetrics for medical articles: An analysis of 45 fields - Thelwall-2016-Journal of the Association for Information Science and Technology_Wiley Online Library. https://asist dl.onlinelibrary.wiley.com/doi/abs/10.1002/asi.23501. Accessed 3 Aug 2020

21. Ahmed S, Gupta L (2020) SOCIAL MEDIA FOR MEDICAL JOURNALS. Cent Asian J Med Hypotheses Ethics 1:25-31. https ://doi.org/10.47316/cajmhe.2020.1.1.04

22. Ahmed S, Gupta L (2020) Perception about social media use by rheumatology journals: survey among the attendees of IRACON 2019. Indian J Rheumatol. https://doi.org/10.4103/injr.injr_15_20

23. Nikiphorou E, Studenic P, Ammitzbøll CG et al (2017) Social media use among young rheumatologists and basic scientists: results of an international survey by the Emerging EULAR Network (EMEUNET). Ann Rheum Dis 76:712-715. https://doi.org/10.1136/annrh eumdis-2016-209718

24. Zimba O, Radchenko O, Strilchuk L (2020) Social media for research, education and practice in rheumatology. Rheumatol Int 40:183-190. https://doi.org/10.1007/s00296-019-04493-4

25. Negrón JB (2019) \#EULAR2018: the annual European congress of rheumatology-a twitter hashtag analysis. Rheumatol Int 39:893-899. https://doi.org/10.1007/s00296-019-04249-0

26. Cho D, Cosimini M, Espinoza J (2017) Podcasting in medical education: a review of the literature. Korean J Med Educ 29:229-239. https://doi.org/10.3946/kjme.2017.69

27. Coronavirus Research Moves Faster Than Medical JournalsBloomberg. https://www.bloomberg.com/opinion/articles/202005-05/coronavirus-research-moves-faster-than-medical-journals. Accessed 3 Aug 2020

28. Samuel H, Zaïane O (2018) MedFact: towards improving veracity of medical information in social media using applied machine learning. In: Bagheri E, Cheung JCK (eds) Advances in artificial intelligence. Springer International Publishing, Cham, pp 108-120

29. Ahmed S (2020) Letter to the editor: social media is a double-edged sword in the COVID-19 pandemic. J Korean Med Sci. https://doi. org/10.3346/jkms.2020.35.e270

30. Pineda C, Pérez-Neri I, Sandoval H (2019) Challenges for social media editors in rheumatology journals: an outlook. Clin Rheumatol 38:1785-1789. https://doi.org/10.1007/s10067-019-04586-2

31. Lahiry S, Choudhury S, Chatterjee S, Hazra A (2019) Impact of social media on academic performance and interpersonal relation: a cross-sectional study among students at a tertiary medical center in East India. J Educ Health Promot 8:73. https://doi.org/10.4103/ jehp.jehp_365_18

Publisher's Note Springer Nature remains neutral with regard to jurisdictional claims in published maps and institutional affiliations. 